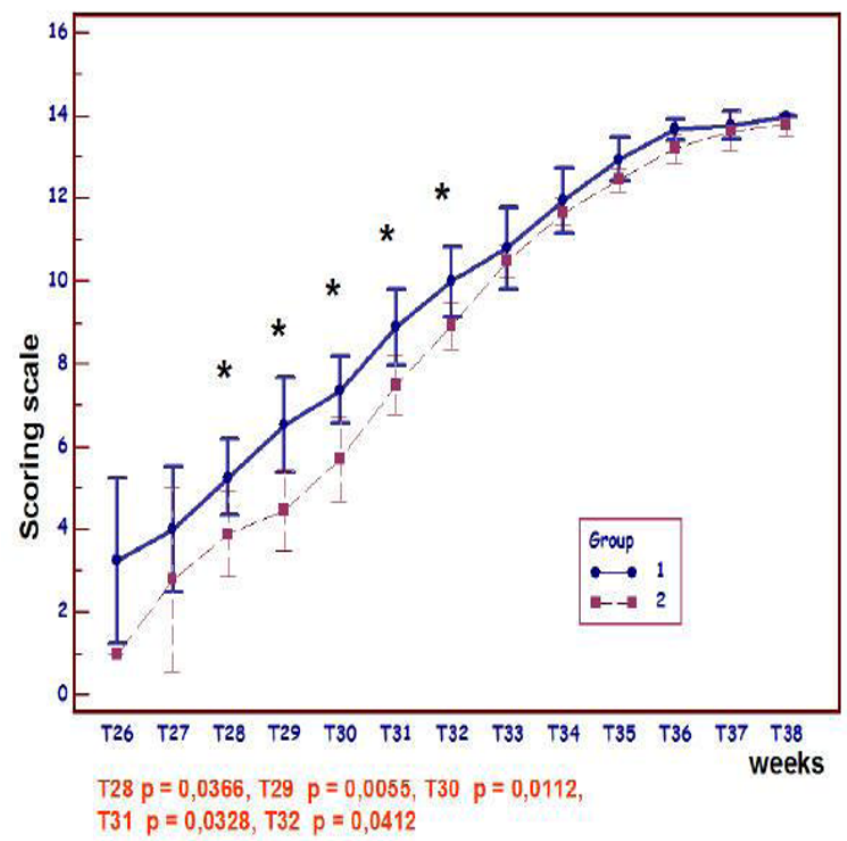

[figure]

Conclusions: Accelerated maturation of cerebral function seems to be associated with long-term cognitive and behavioral deficits without overt effects on motor development in preterm infants who were neurologically normal in the neonatal period.

595

\section{CORRELATION BETWEEN ULTRASOUND TEXTURE ANALYSIS OF THE CEREBELLUM AND STANDARD DIAGNOSIS OF WHITE MATTER DAMAGE IN PRETERM NEONATES}

E. Bonet-Carne ${ }^{1}$, V. Tenorio ${ }^{2,3,4}$, A. Arranz ${ }^{2,3,4}$, I. Amat-Roldan ${ }^{3,4}$, E. Gratacos $2,3,4$

${ }^{1}$ Transmural Biotech, SL, ${ }^{2}$ Maternal-Fetal and Neonatology Departments, Institut Clinic of Gynecology, Obstetrics and Neonatology, Hospital Clinic-Universidad de Barcelona, ${ }^{3}$ Fetal and Perinatal Medicine Research Group, Institut d'Investigacions Biomèdiques August Pi i Sunyer (IDIBAPS), ${ }^{4}$ Centro de Investigación Biomédica en Red de Enfermedades Raras (CIBER-ER), Instituto de Salud Carlos III, Barcelona, Spain

Background and aims: Ultrasound diagnosis of white matter damage (WMD) is still subject to variability, and methods based on quantitative ultrasound analysis are under investigation to improve diagnostic accuracy. The correlation between WMD and cerebellar changes in term and one-year old infants has previously been described. We tested the hypothesis that a semiautomated method for ultrasound texture analysis (AQUA) could detect patterns to predict the standard clinical diagnosis of WMD in preterm neonates.

Methods: A cohort of 40 very preterm infants ( $<32$ weeks) underwent 2 cranial US scans within the first and after 2 weeks of life. WMD was diagnosed using standard criteria. Texture analysis was run in the cerebellum, manually delineated in a coronal plane through the anterior fontanelle. A classification algorithm based on texture coefficients was used to correlate to clinical diagnosis and its ability to predict in the first scan a later diagnosis of WMD was then evaluated.

Results: Median gestational age was $29+4$ weeks $(26+0-31+6)$. WMD was diagnosed in 10/40 infants. Cerebellar texture analysis in the first scan could identify cases later diagnosed with PVL with a sensitivity of $100 \%$ and an accuracy of $95 \%$, after adjustment for gestational age and birth weight.

Conclusions: The cerebellum might contain useful information that improves the prediction of standard white matter damage diagnosis. These preliminary data support further investigation of the role of this brain structure in the neonatal diagnosis of WMD by means of semi-automated quantitative ultrasound analysis.

\section{6}

MRI OF THE BRAIN OF TWINS WITH TWIN TWIN TRANSFUSION SYNDROME. (TTTS)
A. Lyons ${ }^{1}$, M. Boyle 1 , A. Foran', S. Ryan ${ }^{2,3}$
${ }^{1}$ Neonatology, ${ }^{2}$ Radiology, Rotunda Maternity Hospital, ${ }^{3}$ Radiology, Children's University Hospital, Dublin, Ireland

Introduction: Perinatal mortality and morbidity rates are higher in twins than in singletons. Monochorionic twins have a high risk of low birth weight, preterm delivery and neurologic morbidity. TTTS occurs as a serious complication in 10 to $15 \%$ of monochorionic diamniotic (MCDA) pregnancies. The assessment of term corrected MRI brain has not been investigated to the best of our knowledge in this specific area to date.

Objective: The aim of this study was to evaluate term corrected MRI brain as a surrogate short-term marker for neonatal outcome of twins with TTTS.

Study Design: We conducted a prospective trial in the Rotunda hospital which had ethical approval. We 